\title{
Too much of a good thing? Could we be overdoing cerebral protection in neonatal cardiac surgery?
}

\author{
Osman O. Al-Radi, MBBCh, MSc, FRCSC
}

\footnotetext{
From the Department of Surgery, Abdullah Bakhsh Children's Heart Center, King Abdulaziz University, Jeddah, Saudi Arabia; and the Faculty of Medicine, King Abdulaziz University, Jeddah, Saudi Arabia. Disclosures: Author has nothing to disclose with regard to commercial support.

Received for publication Nov 21, 2015; accepted for publication Nov 25, 2015; available ahead of print Jan 9 , 2016.

Address for reprints: Osman O. Al-Radi, MBBCh, MSc, FRCSC, Department of Surgery, Abdullah Bakhsh Children's Heart Center, Faculty of Medicine, King Abdulaziz University, PO Box 80215, Building 10, Room 1027, Jeddah 21589, Saudi Arabia (E-mail: oradi@kau.edu.sa).

J Thorac Cardiovasc Surg 2016;151:1145-6

$0022-5223 / \$ 36.00$

Copyright (C) 2016 by The American Association for Thoracic Surgery

http://dx.doi.org/10.1016/j.jtcvs.2015.11.054
}

The use of deep hypothermic circulatory arrest (DHCA), first introduced by Bigelow and colleagues, ${ }^{1}$ has revolutionized cardiac surgery. Nowhere is this truer than in neonatal cardiac surgery, where DHCA is used liberally for many indications, including but not limited to aortic arch repair and pulmonary vein repair. Not infrequently, it is also used to provide a bloodless field, even without the surgical task requiring circulatory arrest. ${ }^{2}$ The specific parameters of perfusion used in association with DHCA vary somewhat among surgeons. ${ }^{3}$ The lowest temperature, the acid-base management strategy, the lowest hematocrit, and the use of selective cerebral perfusion are among the variables that are often adjusted. The prevailing parameters are perhaps a DHCA lowest temperature of $18^{\circ} \mathrm{C}, \mathrm{pH}$ stat acid-base management, and no antegrade cerebral perfusion (ACP). Cooling and rewarming times are also carefully controlled. The success of DHCA in the immediate outcomes is difficult to challenge. ${ }^{4}$ Postoperative gross neurologic defects are rare, and clinical seizures and abnormal imaging studies are frequent but reversible in the majority of cases. Subclinical seizures are detectable at a higher frequency than observable seizures. Data regarding the longterm effects of DHCA on neurodevelopmental outcomes are scarce. Perhaps the best known study in the subject, the Boston DHCA study, showed that children in whom DHCA was used had higher prevalence of neurologic abnormalities and poorer mental function at 1, 2.5, and 4 years after surgery. 2,5 In a recent study, Algra and colleagues ${ }^{4}$ studied 37 neonates undergoing cardiac surgery with DHCA with or without ACP. Perhaps most interesting was that $50 \%$ of the neonates had preoperative evidence of cerebral injury on magnetic resonance imaging. There was no difference between groups with or without ACP in the development of new cerebral injury. New infarction only occurred with ACP.

It is becoming increasingly clear that the brain is at risk in children with significant congenital heart disease before, during, and after surgery, and perhaps even before and

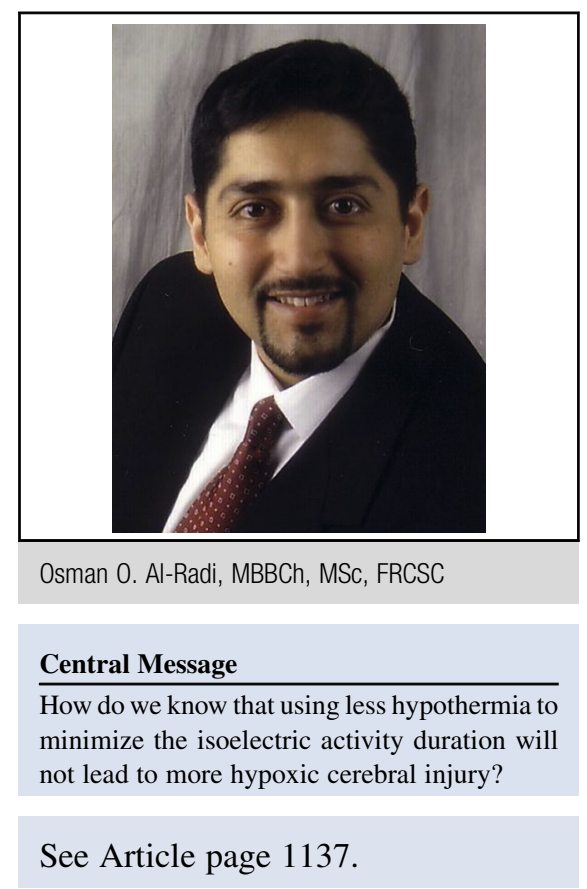

during birth. The finding of new cerebral injury and abnormal early neurodevelopmental parameters may indicate that we are not doing enough to protect the brain during surgery. There is, however, the theoretic possibility that we are doing too much! In this issue of the Journal, Seltzer and colleagues present an analysis of 21 neonates undergoing cardiac surgery with variable diagnoses to assess the correlation between the depth of suppression of brain activity achieved by hypothermia, as measured by continuous intraoperative electroencephalography, and neurodevelopmental outcomes measured by the Vineland Adaptive Behavior Scales-II (VABS-II) at 5 years of age. They found that the duration of the deepest form of brain activity suppression, isoelectric activity (IE), was inversely associated with VABS-II score. Seltzer and colleagues conclude by suggesting that perhaps less suppression of brain activity, less IE, may be less detrimental to the brain and therefore result in better VABS-II scores.

It is important to point out, however, that the patients who had DHCA and therefore longer IE duration versus those who had no DHCA and no or little IE are very different in terms of diagnosis, the complexity of the procedure and likelihood of preoperative cerebral insults. Ten of 11 patients in the IE group had aortic arch hypoplasia and 
coarctation, including 5 who had hypoplastic left heart syndrome. As indicated, there is ample evidence to suggest that such diagnoses are associated with intrauterine and postnatal cerebral events and damage even before surgery. On the other hand, the patients who had no DHCA, 9 of 10 of whom had dextrotransposition of the great arteries, would have been much less susceptible to such risks. A fair comparison would be between patients with similar diagnostic groups and variation only in the level of hypothermia and therefore duration of IE. The analysis presented by Seltzer and colleagues would argue for the counterintuitive point that less IE may be better for the child's neurodevelopmental outcomes. How do we know, however, that if less hypothermia is used to minimize IE duration the brain will not suffer hypoxic injury, which may have much greater effects than a reduction in VABS-II score, such as gross neurologic damage with motor, sensory, or other defects? Until a valid comparison has demonstrated that less hypothermia is indicated, I find it difficult to change my current practice or recommend that to others.

\section{References}

1. Bigelow WG, Lindsay WK, Greenwood WF. Hypothermia: its possible role in cardiac surgery. Ann Thorac Surg. 1950;132:849-66.

2. Newburger JW, Jonas RA, Wernovsky G, Wypij D, Hickey PR, Kuban KC, et al. A comparison of the perioperative neurologic effects of hypothermic circulatory arrest versus low-flow cardiopulmonary bypass in infant heart surgery. $N$ Engl J Med. 1993;329:1057-64.

3. Kouchoukos NT, Karp RB, Blackstone EH, Doty DB, Hanley FL. Hypothermia, circulatory arrest, and cardiopulmonary bypass. In: Kirklin/Barratt-Boyes cardiac surgery. 3rd ed. London: Churchill Livingstone; 2003:66.

4. Algra SO, Jansen NJ, van der Tweel I, Schouten AN, Groenendaal F, Toet M, et al. Neurological injury after neonatal cardiac surgery: a randomized, controlled trial of 2 perfusion techniques. Circulation. 2014;129:224-33.

5. Bellinger DC, Wypij D, Kuban KC, Rappaport LA, Hickey PR, Wernovsky G, et al. Developmental and neurological status of children at 4 years of age after heart surgery with hypothermic circulatory arrest or low flow cardiopulmonary bypass. Circulation. 1999;100:526-32. 